\title{
Modeling of Total Thruster Performance for NASA's Evolutionary Xenon Thruster Ion Optics
}

\author{
Jerold W. Emhoff* \\ Johns Hopkins University Applied Physics Laboratory, Laurel, Maryland 20723 \\ and \\ Iain D. Boyd \\ University of Michigan, Ann Arbor, Michigan 48109
}

\begin{abstract}
An axisymmetric ion-optics model is applied to NASA's Evolutionary Xenon Thruster (NEXT) ion engine. The model is used to simulate the performance of the entire thruster by modeling several apertures at varying radii on the thruster face and integrating the results. The integrated results are compared to experimentally measured data for the NEXT thruster, showing good agreement in most areas. The primary area of discrepancy is in the accelerator grid current, although erosion results suggest that the measured current is unaccountably high. The model is also used to estimate the life of the thruster before the onset of electron backstreaming. Three separate methods are applied, and each predicts thruster failure after approximately 40,000 hours of operation or $845 \mathrm{~kg}$ of xenon throughput.
\end{abstract}

\section{Introduction}

$\mathbf{N}$ ASA's Evolutionary Xenon Thruster (NEXT) is the successor to the very successful NASA Solar Technology Application Readiness (NSTAR) ion engine. ${ }^{1}$ NEXT has a $40-\mathrm{cm}$ beam diameter, compared to NSTAR's 30-cm diam. NEXT has undergone a 2000-h wear test, from which experimental results for performance and thruster life have been obtained. ${ }^{2,3}$ The NEXT thruster used in the life test had a 40-cm beam diameter compared to a 30-cm diam for the NSTAR thruster. The thruster also has an improved discharge chamber design, resulting in a flatter current density profile across the thruster face. ${ }^{4}$

Simulation of ion optics to date have focused primarily on accurately modeling the performance of the ion optics and the life of the thruster. Several three-dimensional models are currently in development. Malone and Soulas ${ }^{5}$ and Anderson et al. ${ }^{6}$ make use of a gun-type model. Farnell et al. ${ }^{7}$ have developed a model similar to a gun simulation, but with the important inclusion of self-consistent sheath formation. Three-dimensional particle-in-cell models are applied by Kafafy and Wang ${ }^{8}$ and Okawa et al. ${ }^{9}$ The use of a full three-dimensional model allows accurate simulation of erosion on the downstream face of the accelerator grid as well as better comparison to experimental measurements.

Unlike these, the model presented in this work is an axisymmetric simulation of a single ion-optics aperture. Although this model cannot predict erosion of the accelerator grid downstream face accurately, it has features that set it apart from any of the threedimensional models. One such feature is the modeling of the neutral gas in the ion optics via direct particle simulation, rather than the fluid model used by most other simulations. The model also has the ability to simulate sputtered grid material, as well as the recombination of that material with ion-optics surfaces.

Presented as Paper 2005-3687 at the AIAA/ASME/SAE/ASEE Joint Propulsion Conference and Exhibit, Tucson, AZ, 10-13 July 2005; received 20 July 2005; accepted for publication 18 November 2005. Copyright (C) 2006 by the American Institute of Aeronautics and Astronautics, Inc. All rights reserved. Copies of this paper may be made for personal or internal use, on condition that the copier pay the $\$ 10.00$ per-copy fee to the Copyright Clearance Center, Inc., 222 Rosewood Drive, Danvers, MA 01923; include the code 0748-4658/06 \$10.00 in correspondence with the CCC.

*Postdoctoral Researcher, Aeronautical Science and Technology Group, Research and Technology Development Center, 11100 Johns Hopkins Road; Jerold.Emhoff@ jhuapl.edu. Member AIAA.

${ }^{\dagger}$ Professor, Aerospace Engineering Department, 1320 Beal Avenue; iainboyd@engin.umich.edu. Associate Fellow AIAA.
This model has been under development for several years ${ }^{10}$ and has previously been applied to both the NSTAR ${ }^{11}$ and NEXT ${ }^{12-14}$ ion engines. The simulation is applied to NEXT in this work, with the goal of comparing simulated performance and erosion to experimental measurements. This is accomplished by simulating several apertures across the thruster face that operate at different beamlet currents and geometrical conditions. The results from these simulations are then integrated to obtain performance for the entire thruster. Three methods are also used to estimate the life of the thruster before failure caused by electron backstreaming: 1) initial erosion characteristics are calculated and assumed to remain constant to determine life; 2) multiple simulations are performed, between which a fixed amount of erosion is allowed to occur to determine life; and 3) dynamic (continuous) simulations are performed to determine life.

The paper first briefly discusses the model operation and operating conditions. Next, the methodology and results for total thruster simulation are given, including a discussion of the integration procedure, comparison to experimental data, and discussion of the accelerator grid current. A brief study of electron backstreaming is given, followed by simulation results for thruster life.

\section{Model Operation}

The computational model simulates a single two-dimensional axisymmetric aperture in an ion thruster. A computational mesh composed of evenly spaced rectangular cells is used to track particles in the simulation. The optics of the thruster are simulated using boundary cells in the domain. These cells can be arranged in an irregular way, allowing the simulation of cusps on the barrels of the aperture. Figure 1 shows a typical domain, with the top half of the plot showing the meshed representation, and the bottom half showing the actual geometry. The radius of the domain is set to half the center-to-center spacing between adjacent apertures in the ion optics.

The code uses the particle-in-cell ${ }^{15}$ (PIC) method to simulate doubly and singly charged xenon ions and xenon neutrals. Each computational particle has a numerical weight that indicates the actual number of atoms represented by the particle. Flowfield quantities for each cell are obtained by averaging the properties of all of the particles in the cell, taking into account the weight of each particle. The potential field accelerates ions self-consistently, and electrons are modeled as a fluid via the Boltzmann relation. The direct-simulation Monte Carlo method ${ }^{16}$ is used for processing particle collisions. Both charge-exchange (CEX) and momentum-exchange collision types are simulated. Dalgarno et al. ${ }^{17}$ momentum-exchange collision 


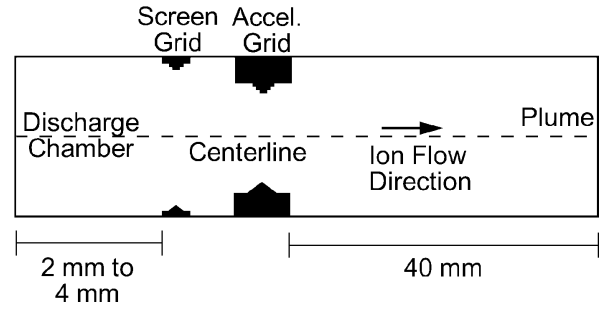

Fig. 1 Plot of the simulation domain. The upper half is the meshed simulation domain, whereas the bottom half is the actual domain geometry.

cross sections with isotropic scattering are used for simulating momentum-exchange collisions. On the order of 100,000 time steps are performed to reach a steady-state of ion flow, after which results are sampled for another 100,000 time steps.

The sputter yield for xenon impacting on molybdenum is determined in the simulation by two models. The first model is a curve fit to experimental data giving the energy dependence of the sputter yield $^{18,19}$ for impacts normal to the surface. The second model gives the relative yield as a function of the angle of impact for $300-\mathrm{eV}$ particles. The calculated results from these two models are multiplied to obtain the total sputter yield for a given impact. This assumes that the relative angular yield does not vary greatly with impact energy.

The nominal simulation geometry is that of the NEXT ion optics, except where noted otherwise. The grid gap used is the cold grid gap of the thruster, which is about $80 \%$ of the accelerator grid thickness. The hot grid gap of the thruster might in reality be much smaller than this.

The conditions simulated for the NEXT thruster are set to the maximum power operating point of the thruster, and the beamlet on the thruster centerline is usually modeled. This involves a discharge potential of $1800 \mathrm{~V}$, a screen grid potential of $1776 \mathrm{~V}$, and an accelerator grid potential of $-210 \mathrm{~V}$. The beamlet current for the centerline aperture is approximately $0.168 \mathrm{~mA}$, based on the peak experimental current density.

The plume plasma potential is set to $22 \mathrm{~V}$ in most cases, although the experimentally measured plasma potential is about $16 \mathrm{~V}$ (Ref. 3). This discrepancy does not greatly affect most results, with the exception of the electron backflow current calculation. The only effect is simply that the potential plateau downstream of the plume sheath is $6 \mathrm{~V}$ higher. This reduces the total accelerating potential by less than $1 \%$, so that the effect on the thrust and beamlet current is negligible. The electron backflow current is affected more strongly because this calculation depends exponentially on the plume plasma potential via the Boltzmann relation.

\section{Total Thruster Simulation}

Performance data have been measured for the NEXT ion engine during a 2000-h wear test. ${ }^{20}$ To obtain accurate computational results for comparison to this performance data, it might be necessary to simulate several apertures at varying radii on the thruster optics. The results from these simulations can then be integrated to give results for the entire thruster. However, because the ion current determines much of the performance of the thruster it is possible to estimate the thruster performance by scaling results from a single aperture simulation to all apertures, based on the beamlet current.

\section{Beam Current Density Profile Scaling}

A beam current density profile ${ }^{2}$ for the NEXT ion engine operating at $3.52 \mathrm{~A}$ is plotted in Fig. 2. This profile can be used to determine the radius of a given single-aperture simulation by matching the beamlet current density at the downstream edge of the simulation domain to the profile. It can also be used to integrate thruster performance based on a single simulation. However, the experimental current density profile presents two problems. First, the profile extends beyond the beam extraction area of the thruster. This is resolved by scaling the radius by a factor of approximately 0.988 at each point, such that the second-to-last point on the profile is at

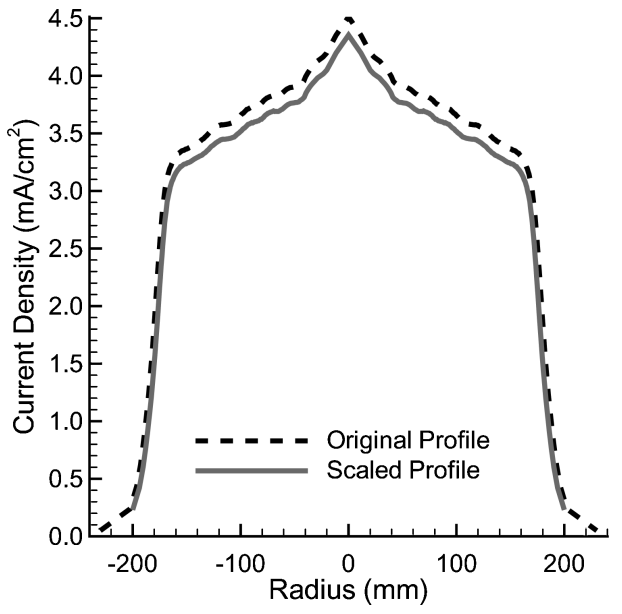

Fig. 2 Experimentally measured and scaled beam current density profiles for the NEXT ion engine extracting 3.52 A of beam current. The scaled profile ignores the outermost experimental point, then scales the radius of the remaining points to be within $\pm 200 \mathrm{~mm}$. The radius-scaled profile is then scaled such that the integral of the profile gives the correct beam current.

$\pm 20 \mathrm{~cm}$. The last point is ignored completely, as it is far from any of the other measured points and is well beyond the beam diameter of the thruster.

Second, when integrated, the profile gives a beam current higher than that of the thruster. This is accounted for by scaling the profile down such that integrating it gives the correct beam current. This scaling factor is about 0.965 at the 3.52-A beam current operating point. The profile resulting from these two scaling methods has a flatness parameter of about 0.71 , the same as is measured experimentally. This indicates that the scaled profile is reasonably accurate for the purposes of locating simulated apertures on the thruster. The scaled version of the profile is also shown in Fig. 2.

\section{Multiple-Aperture Simulation Results}

The multiple-aperture simulation results are performed with varying aperture diameters. The ion optics used in the NEXT 2000-h wear test have decreasing aperture diameters as the thruster radius increases, as a result of the grid manufacturing process. To obtain accurate results, the aperture diameters are varied in the simulations as well.

In each simulation, the upstream domain length is set to $2 \mathrm{~mm}$ for all but the lowest current case. For this case, the domain length is set to $4 \mathrm{~mm}$. The downstream domain length is $4 \mathrm{~cm}$ in each case. These lengths have been determined as sufficient in previous work. ${ }^{14}$ Sputtered grid material is not modeled in any of the simulations.

Figure 3 shows the variation of both the accelerator and screen grid aperture diameters as a function of radius, normalized to the nominal centerline aperture diameter. ${ }^{2}$ Also shown are the simulated aperture locations and diameters. The experimental grid diameters were measured using a pin gauge; thus, the aperture diameters can be up to $0.0254 \mathrm{~mm}$ larger in reality. The simulations use a constant radial cell size, so that there is some error in the representation of the aperture diameters and cusp structures. Also, when a simulation is run, the exact output beamlet current is not known beforehand, and so an estimate of the radius on the thruster is required in order to determine what the aperture diameters will be. However, the aperture diameters change very rapidly for midrange thruster radii, and the beamlet current changes very slowly. The result is that a small change in beamlet current can produce a large change in aperture diameter. This explains the discrepancies seen in the plot.

The computed thrust, beamlet current, mass flow rate, and accelerator grid current for a single aperture are shown in Fig. 4 as a function of radius. All quantities are normalized to the centerline value. The thrust varies nearly linearly with the beamlet current, with less than $3 \%$ difference in Fig. 4, as the beam ions produce almost all of the thrust. The mass flow rate is nearly linear with 


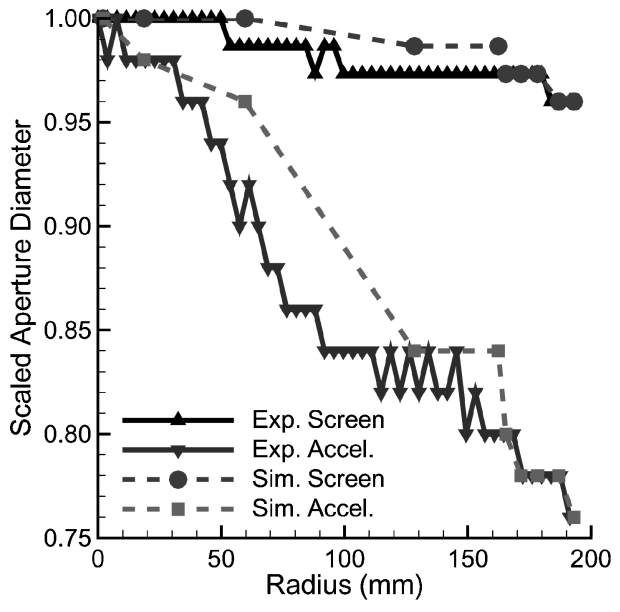

Fig. 3 Variation of the accelerator and screen grid aperture diameters as a function of radius on the thruster. The simulated aperture diameters are also shown.

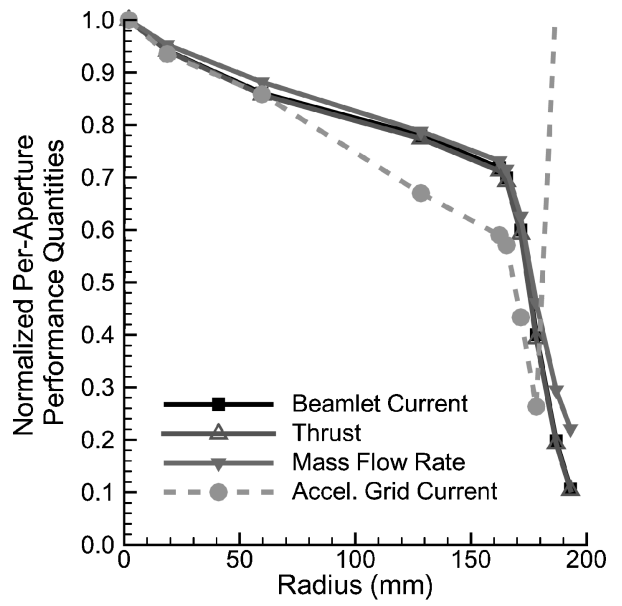

Fig. 4 Variation of simulated per-aperture performance quantities as a function of radius. Each quantity is normalized by the centerline value.

the beamlet current, but at low currents the mass flow rate does not drop as quickly as the beamlet current. This is because the discharge neutral density is held constant across all apertures, and so the total flow rate does not drop as quickly when the ion flow rate is small.

The accelerator grid current decreases more quickly as the radius increases than the beamlet current or thrust. This is caused by the decrease in screen and accelerator grid aperture diameters. As the diameter decreases, fewer neutrals from the discharge chamber are able to flow through the optics, leading to a lower neutral density downstream of the ion optics. The CEX production rate is a linear function of both ion and neutral density, so that, as the neutral density decreases, the accelerator grid current will decrease as well. Also, the two simulated apertures at the highest radii have much larger accelerator grid currents than the other apertures. The low beamlet current in both cases induces crossover and direct impingement of beam ions on the accelerator grid barrel. This direct impingement will in reality quickly erode the aperture wall until the beamlet no longer impinges directly.

\section{Integration Procedure}

The multiple-aperture simulation results are integrated to obtain total thruster performance results. Each relevant quantity, for example thrust or beamlet current, obtained for an individual aperture is divided by the simulation area in order to obtain a density value instead. The density value is then assumed to vary linearly between the simulated apertures. The resulting linear approximating function is integrated between the points and over the thruster surface area to give the contribution between the points. The individual contri- butions are then summed over all points to obtain the total thruster performance quantities. In equation form, the process is as follows for a quantity $Q$ :

$$
\begin{gathered}
q_{i}=\frac{Q_{i}}{A_{\text {sim }}}=a_{i} r_{i}+b_{i} \\
a_{i}=\frac{q_{i}-q_{i+1}}{r_{i}-r_{i+1}} \\
b_{i}=-a_{i} r_{i}+q_{i} \\
Q_{\text {Total }}=\pi \sum_{i=1}^{N-1} \frac{2 a_{i}}{3}\left(r_{i+1}^{3}-r_{i}^{3}\right)+b_{i}\left(r_{i+1}^{2}-r_{i}^{2}\right)
\end{gathered}
$$

Here $a_{i}$ and $b_{i}$ are the slopes and intercepts of the linear approximation functions, respectively; $A_{\text {sim }}$ is the simulated aperture domain area; and $N$ is the number of simulated points.

To approximate the total thruster performance using a single aperture, the quantities of interest are scaled according to the beam current density profile in order to obtain values at all points. The scaled values are then integrated in the same way as shown in order to obtain total thruster performance results. This procedure will give the best results when the quantity of interest varies linearly with the beamlet current. The centerline beamlet current simulation is used in these cases as the base, but any simulation can be used. The values from the simulation are simply scaled up or down depending on the simulated aperture's position on the current density profile.

Note that not all quantities can scale with the beamlet current, for example, the screen grid ion transparency. In most cases however, the quantity can be determined for the entire thruster by using other means. In the case of the screen grid ion transparency, this can be determined simply by dividing the integrated beam current with the sum of the integrated screen grid and beam currents. For other quantities, such as electron backstreaming current, multiple simulations might be required.

\section{Total Thruster Performance Results}

In Table 1, integrated simulation performance results are compared to experimental data. Both multiple-aperture and singleaperture simulation performance results are shown. As the table shows, the multiple-aperture integration gives excellent comparison to the experimental results in most cases. The thrust has less than $1 \%$ error, the mass flow rate is high by only $2.4 \%$, and the specific impulse is within $2 \%$ of the experimental value. The mass flow rate is higher because of a slightly high neutral flow rate imposed in the simulations. Note that the experimental thrust is actually a calculated value, as the thrust is not measured directly.

The screen grid current is higher than the experimental value by less than $6 \%$. The difference seen here might be caused by the inaccuracy of the simulation of the cusp structure on the screen grid aperture wall. Also, the accelerator grid current is about three times smaller than the experimental data. The beam current should be very close to the experimental value, as the experimental current density profile is used to determine the aperture radii. Thus, any error in the beam current integration will induce integration error

Table 1 Comparison of performance quantities for multipleaperture simulations and single-aperture integration against experimental data from the NEXT ion engine 2000-h wear test ${ }^{2}$

\begin{tabular}{lccc}
\hline \hline Quantity & $\begin{array}{c}\text { Experimental } \\
\text { data }^{2}\end{array}$ & $\begin{array}{c}\text { Multiple } \\
\text { simulation }\end{array}$ & $\begin{array}{c}\text { Single } \\
\text { simulation }\end{array}$ \\
\hline Mass flow rate, mg/s & 5.87 & 6.01 & 6.24 \\
Thrust, mN & 237 & 239 & 241 \\
Specific impulse, s & 4117 & 4055 & 4001 \\
Beam current, A & 3.52 & 3.51 & 3.52 \\
Screen grid current, A & 0.44 & 0.465 & 0.462 \\
Accelerator grid current, mA & 12.5 & 4.77 & 3.88 \\
Erosion rate, mg/k-h & - & 26.46 & 5.64 \\
\hline \hline
\end{tabular}


for all quantities. Also, the beam current for the single-simulation case should be exact, as the beam current density curve is simply integrated to obtain the value.

The single-aperture integration results do not compare as well as the multiple-aperture results. This is expected, especially given that quantities such as mass flow rate and accelerator grid current do not vary linearly with the beamlet current. However, there is still excellent prediction of thrust and screen grid current. There is some difference from the multiple-aperture results in accelerator grid current and erosion rate, much of which is caused by the direct impingement seen in the outer, low-current apertures.

The simulated screen grid currents listed in Table 1 are corrected values. The current actually simulated by the model is approximately $0.13 \mathrm{~A}$ in each case. However, the axisymmetry of the simulation does not account for the hexagonal arrangement of the thruster apertures. The screen grid current is corrected for ions collected on the areas of the screen grid that are not simulated. The correction to the current is about twice as large as the simulated amount of current. In Table 1, approximately $30 \%$ of the current is simulated, while the corrected current provides the remaining $70 \%$.

\section{Accelerator Grid Current Discussion}

The property offering the poorest comparison between simulation and experiment, for both multiple- and single-aperture integration, is the accelerator grid current. In both cases, the simulated accelerator grid current is low by a factor of about 3 . The simulation domain downstream of the ion optics is sufficient to collect a converged amount of CEX ion current, and so there is no current deficiency caused by a short domain. No facility backpressure is simulated; however, the tank pressure is low enough that it does not significantly increase the neutral density in the downstream region. This can be seen in Soulas et al., ${ }^{2}$ as the measured accelerator grid current did not significantly increase when the tank pressure increased during the 2000-h wear test. The deficit in current should be seen in the erosion rate as well, as both depend on CEX ions. If the simulated erosion rate is three times too low as well, then the simulation is clearly operating incorrectly.

Figure 5 shows the increase in accelerator grid aperture diameters following $2000 \mathrm{~h}$ of thruster operation. Experimentally measured pin gauge results and simulation results are plotted. Also shown are the aperture diameters assuming an erosion rate three times higher than is simulated, representing a first-order estimate of the erosion from the simulation for an accelerator grid current similar to the measured value. The erosion in the simulations compares well to the experimental data, as it is generally higher than the measured value by approximately $0.0254 \mathrm{~mm}$ or less. There is more

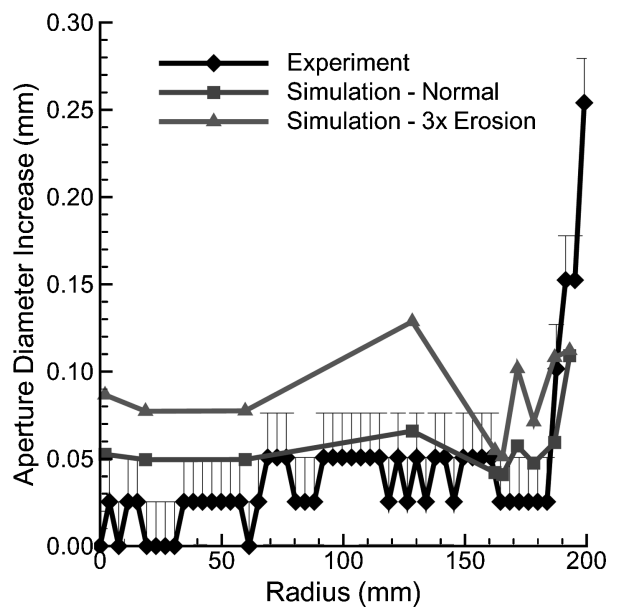

Fig. 5 Aperture diameter increase as a result of erosion after $2000 \mathrm{~h}$ of thruster operation. The pin-gauge measured experimental data and the simulation results are shown. Results for tripled erosion rates are plotted as well. Crossover of the beamlet in the low-current apertures at high radii causes the large amount of erosion seen in these apertures. error for apertures near the thruster edge because these apertures experience direct impingement as a result of beamlet crossover, as well as noncircular erosion caused by misalignment of the screen and accelerator grid apertures. The axisymmetric simulation is unable to accurately model this type of erosion.

The unaltered erosion matches the experimental data well, whereas the tripled erosion rate results are much higher than the experimentally measured values. This indicates that the barrel erosion rate is approximately correct, and thus the current collected on the barrel can be assumed to be correct as well. If the simulation is at fault for the deficient accelerator grid current, this current must be collected on the downstream face of the grid.

Consider the centerline aperture only. The experimental current collected from this aperture should be approximately $0.61 \mu \mathrm{A}$. The simulated barrel current for this aperture is $0.081 \mu \mathrm{A}$, leaving $0.53 \mu \mathrm{A}$ of current to be collected on the downstream face. Assuming that all ions impact the grid surface at $230 \mathrm{eV}$ and normal incidence, this amount of current will erode approximately $1.4 \mathrm{mg}$ of grid material over $2000 \mathrm{~h}$. An estimate of the eroded mass seen in the 2000-h wear test of the NEXT thruster can be made based on profilometer measurements. ${ }^{3}$ This estimate gives $0.47 \mathrm{mg}$ of eroded grid material around a single aperture, one-third the value calculated based on the measured current. The simulation gives $0.25 \mathrm{mg}$ of eroded material after $2000 \mathrm{~h}$ of erosion, or half the estimated value. These erosion values are summarized here: inferred from measured accelerator grid current-1.4 mg; inferred from profilometry data- $0.47 \mathrm{mg}$; and simulation- $0.25 \mathrm{mg}$.

The erosion inferred from the measured accelerator grid current just given, and the simulated erosion on the downstream face, do not take into account reduced erosion caused by the pit-and-groove structures. As these form, eroded material is more likely to recombine on the wall of the pit or groove because the viewing angle of the sputtered material with respect to the grid walls is increased. In other words, assuming that sputtered material leaves the wall at a random angle, the range of angles that will result in recombination on the grid surface increases as the depth of the pit or groove increases. Also, the angle of incidence of impacting ions will increase as the walls of the pit or groove become steeper, reducing the sputter yield. However, it is not likely that this accounts for three times less erosion than the amount inferred from the measured accelerator grid current. The pit-and-groove structures are not deep after $2000 \mathrm{~h}$ of testing, and so any effect of either ion incidence or sputtered material viewing angle that might reduce the net erosion is small.

Another possible effect is caused by uncertainty in the sputter yields. The sputtering model used here gives a yield of 0.37 atoms of sputtered material per $230-\mathrm{eV}$ ion, and this is consistent with most experimental sputtering data. ${ }^{21-24}$ However, data collected by Doerner et al. ${ }^{25}$ indicate that the sputter yield is a much lower 0.1 atoms per ion. If this sputter yield were used instead, the amount of inferred erosion would instead be $0.38 \mathrm{mg}$, which is slightly lower than the profilometry-based estimate of $0.47 \mathrm{mg}$. The sputter yield in a vacuum facility can also be reduced by carbon deposited on the grid surface caused by backsputtering of the beam targets in the vacuum chamber.

The simulation underestimates the amount of erosion somewhat, but there is still a large discrepancy between the erosion values inferred from the measurements of accelerator grid current and profilometry.This suggests that some portion of the measured accelerator grid current originates from a location other than around the ion optics apertures. One such location could be the outer rim of the accelerator grid, although no experimental erosion has been observed in this region. Another possibility is secondary electron emission from the accelerator grid, which would increase the measured current. This effect is expected to be negligible in ion thruster optics however.

A three-dimensional simulation of the downstream accelerator grid face erosion is also required for further study, as the present model cannot reproduce the pit-and-groove structures. If the downstream face erosion in a three-dimensional simulation matches the experimental data and the accelerator grid current is still deficient, then the experimental current is being collected at a point that is not 
intended to be simulated. Three-dimensional simulations of downstream face erosion have been performed by Farnell et al. ${ }^{7}$ and Wang et al., ${ }^{26}$ but it is unknown if the accelerator grid current was deficient in these cases.

\section{Electron Backstreaming Study}

One of the primary failure modes of an ion thruster is loss of performance as a result of electron backstreaming. This occurs when the accelerator grid aperture diameter increases as a result of erosion, such that there is no longer a retarding potential keeping plume electrons from being accelerated into the discharge chamber. This form of engine failure can be mitigated by increasing the magnitude of the potential on the accelerator grid; however, this also has the effect of accelerating the erosion, as impacting ions will have a higher energy.

The onset of electron backstreaming occurs when the minimum centerline potential in the ion optics rises to a point such that electrons are able to backstream. The centerline will in all likelihood have the highest potential, as it is furthest from the accelerator grid, and also because the ion density is generally highest on the centerline. This is true for the range of beamlet currents encountered during nominal operation of the NEXT thruster.

The electron backstreaming limit in the NEXT ion engine has previously been measured experimentally. ${ }^{2,4}$ The backstreaming limit for a given ion-optics geometry is measured by decreasing the accelerator grid potential magnitude until the measured beam current increases by $0.1 \mathrm{~mA}$. Assuming that the current is uniform for all apertures, this is approximately $3.36 \times 10^{-9} \mathrm{~A}$ of electron current for a single aperture. This assumption is not true, as apertures near the centerline of the thruster will generally produce backstreaming before the other apertures, because the higher ion density and larger aperture diameters result in a less negative minimum centerline potential. Thus, the backstreaming current will be higher near the centerline of the thruster, and an aperture at this location can be expected to have a backflow current higher than $3.36 \times 10^{-9} \mathrm{~A}$. However, the variation in the backflow current with radius is unknown and can be complex, so that the value of $3.36 \times 10^{-9} \mathrm{~A}$ is used as the limit here. The centerline aperture is simulated in the following cases as it will produce the most electron backflow current.

Figure 6 shows the simulated minimum centerline potential as a function of accelerator grid potential. Also shown is the plume plasma potential of $22 \mathrm{~V}$ and the experimentally measured backstreaming potential of $-172 \mathrm{~V}$. The plot shows that the minimum centerline potential at the experimentally measured backstreaming limit is about 7.5 V. For the simulation, this is then the point at which electron backstreaming is assumed to occur.

For the simulated plume plasma potential of $22 \mathrm{~V}$ and electron temperature of $1 \mathrm{eV}$, the single-aperture electron backflow current at

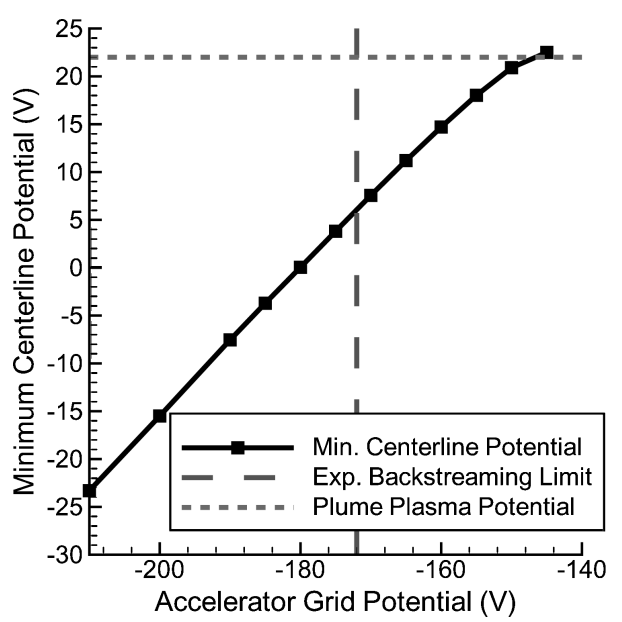

Fig. 6 Minimum centerline potential as a function of accelerator grid potential. The downstream plasma potential and experimental backstreaming potential are also shown. this point is estimated to be $4 \times 10^{-13} \mathrm{~A}$, several orders of magnitude lower than the $3.36 \times 10^{-9}$ A limit used experimentally. However, experimental measurements ${ }^{3}$ indicate that the plume plasma potential is approximately $16 \mathrm{~V}$, not $22 \mathrm{~V}$. If a $16-\mathrm{V}$ plume potential is assumed, then the electron backflow current will be $8.8 \times 10^{-11} \mathrm{~A}$, somewhat closer to the experimental limiting value, although still two orders of magnitude too low. The electron current is also very sensitive to the electron temperature. ${ }^{14}$ An increase to $1.5 \mathrm{eV}$ is sufficient to reach the experimental electron current limit for a $16-\mathrm{V}$ plume potential. However, the experimentally measured plume electron temperature is approximately $1 \mathrm{eV}$ (Ref. 3), the same as nominally simulated, so that such an increase is not likely. The minimum centerline potential is not affected strongly by the electron population properties, so that the $7.5-\mathrm{V}$ potential is used as the indicator of electron backstreaming, rather than the electron backflow current itself.

Although the electron backstreaming limit is found experimentally by varying the accelerator grid potential, the thruster will actually fail when the accelerator grid aperture diameters increase enough such that backstreaming occurs. For simulations where the aperture is eroded until thruster failure, it is more useful to know at what aperture diameter electron backstreaming will occur.

To determine the necessary aperture diameter to allow electron backstreaming, several simulations are performed with a gradually increasing diameter while the grid potential is held constant at $-210 \mathrm{~V}$. In each case, one layer of cells is removed from the accelerator grid barrel. Figure 7 shows the resulting minimum centerline potential as a function of aperture diameter. The diameters here are normalized by the nominal aperture diameter. Electron backstreaming occurs when the aperture enlarges by approximately $35 \%$.

A point to note here is that the minimum centerline potential increases in a quadratic manner as the aperture diameter is increased. This is most interesting because the area of the aperture wall increases with the square of the diameter. So, the aperture diameter will increase with the square root of time, given a constant erosion rate. However, Fig. 7 indicates that the two effects will cancel, resulting in a linear increase in minimum centerline potential with time. This is important because at a first glance it might appear that the increasing aperture area will slow the approach to electron backstreaming to a less than linear rate.

This method of aperture enlargement does not account for reduction in the thickness of the grid and also assumes that the aperture diameter is increasing uniformly. A thinner grid will allow backstreaming to occur sooner, and a nonuniform erosion pattern might do this as well. The downstream face of the accelerator grid generally forms pits and grooves rather than thinning uniformly however, so that the assumption of a constant grid thickness over time will not likely have a strong effect on the centerline potential of the aperture.

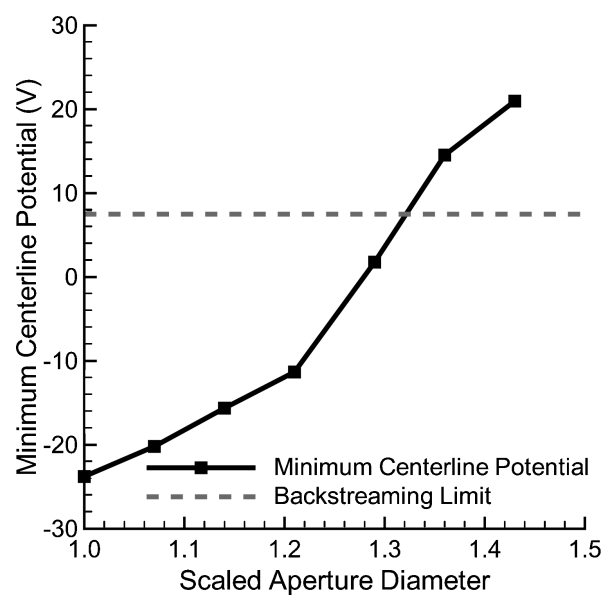

Fig. 7 Minimum centerline potential as a function of accelerator grid aperture diameter. Also shown is the $7.5-\mathrm{V}$ potential at which electron backstreaming will occur. The accelerator grid potential is $-210 \mathrm{~V}$ for these cases. 


\section{Thruster Life Modeling Results}

Thruster failure as a result of electron backstreaming can be estimated by the model in several ways. A single simulation gives results for the erosion rate in each accelerator grid cell. These erosion rates can be used to erode the ion optics until the aperture is large enough to allow electron backstreaming. Another method is to use the erosion rates from the nominal geometry to erode over a set amount of time. Then a new simulation is run with the eroded geometry, and the erosion rates from the second simulation are used to erode the grid further. This process is continued until electron backstreaming occurs. Finally, a dynamic erosion algorithm can be used to erode the ion optics during a single simulation.

The axisymmetric domain of the model does not allow for accurate simulation of erosion on the downstream face of the ion optics. The hexagonal arrangement of the apertures in the optics creates a pit-and-groove pattern, where pits form at the center point between three adjacent apertures and grooves or lines form between adjacent pits. The simulation is unable to model either of these structures. Thus, estimation of the life of the ion optics before they incur structural failure is not possible with the current model, and erosion of the downstream face is ignored in the following study.

\section{Multiple and Single Simulation Results}

The thruster life is first estimated using multiple simulations with static geometry. In each case, the cusped NEXT ion-optics wear test geometry is simulated at the $3.52-\mathrm{A}, 1800-\mathrm{V}$ operating point. The peak beamlet current of approximately $0.168 \mathrm{~mA}$ is modeled, and the accelerator grid potential is fixed at $-210 \mathrm{~V}$. Five simulations are performed in total, with $10,000 \mathrm{~h}$ of erosion being simulated at each step. The initial geometry is simulated first, which gives erosion rates on the accelerator grid barrel. A different erosion rate is obtained for each axial cell on the surface of the aperture wall, so that the surface does not erode uniformly. These erosion rates are applied to the geometry to erode it for $10,000 \mathrm{~h}$. The $10 \mathrm{k}-\mathrm{h}$ erosion case is then simulated to give updated erosion rates, which are used to erode to $20 \mathrm{k}-\mathrm{h}$. This is done until electron backstreaming occurs after approximately $40 \mathrm{k}$-h of erosion. At each step, the entire flowfield is updated, not just the grid geometry. This ensures that the evolution of the erosion pattern is as accurate as possible.

For each column of simulated optics cells, the erosion rate is applied to the cell with the smallest radius. Erosion then occurs in a strictly radial fashion, and downstream face erosion is not accounted for. Also, because the erosion simulated on the downstream corner of the grid includes erosion of the downstream face, the erosion for that cell is always set to be the same as for the cell directly upstream of it.

The ion-optics geometry at each simulation step is shown in Fig. 8 . Very little erosion occurs on the upstream surface of the grid, and

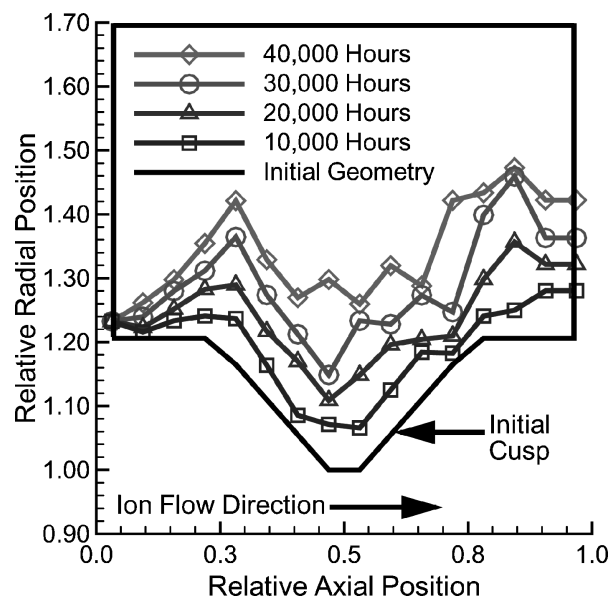

Fig. 8 Geometry of the accelerator grid barrel as a function of erosion time. The axial position is normalized by the grid thickness, with its origin at the grid upstream surface. The radial position is normalized by the initial aperture radius- the origin is on the domain centerline.

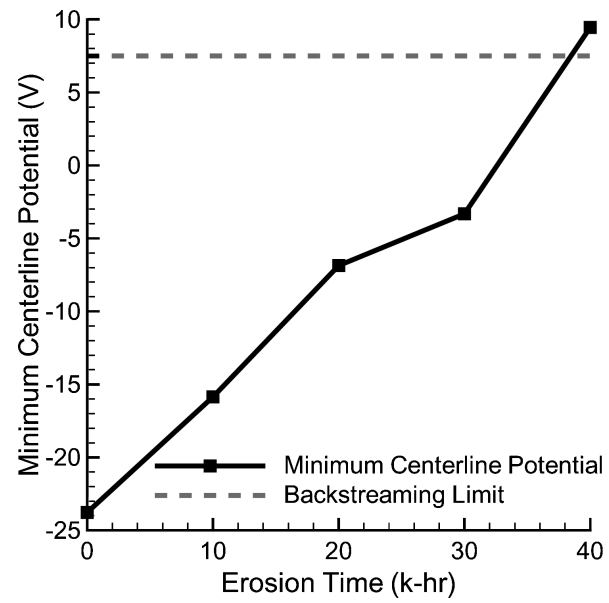

Fig. 9 Minimum centerline potential as a function of erosion time. The backstreaming limit is also shown. The potential varies linearly with time, indicating that backstreaming may be predicted based solely on initial erosion rates.

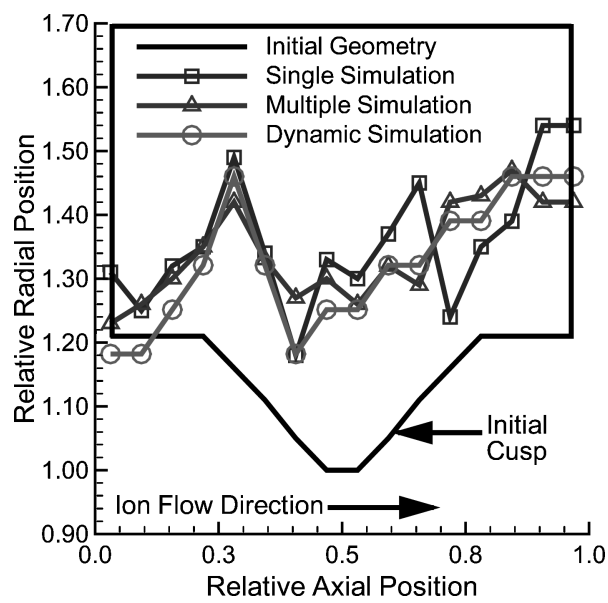

Fig. 10 End-of-life accelerator grid barrel geometry for single, multiple, and dynamic simulations. The single- and multiple-simulation final geometries are both reached after approximately $40,000 \mathrm{~h}$ of erosion. The multiple-simulation case is generally smoother because erosion is redistributed as different parts of the grid are eroded. The final dynamic erosion geometry is reached after $45,000 \mathrm{~h}$ of erosion.

so these points do not vary greatly over the life of the thruster. The erosion rate then increases towards the center of the grid, creating a recess in front of the grid midpoint. This recess is caused by high-energy CEX ions created in the intergrid region at a relatively high potential, which then flow from their point of creation and accelerate to the grid at the recess location. Erosion in the center region is average, followed by another region of high erosion. The erosion is higher on the downstream half of the grid as a result of collection of CEX ions created near the accelerator grid aperture and in the downstream region.

The minimum centerline potential as a function of erosion time is plotted in Fig. 9. As the plot shows, the electron backstreaming limit is reached shortly before $40 \mathrm{k}-\mathrm{h}$ of erosion. The increase in potential is approximately linear with time as well, which indicates that the backstreaming behavior can be predicted easily given initial erosion rates. This linear increase is also consistent with the results given in the section on electron backstreaming.

The life of the thruster can also be estimated by eroding the accelerator grid barrel using only erosion rates simulated at the initial geometry. The results plotted in Fig. 9 indicate that this method is also effective. Indeed, electron backstreaming is found to occur in this case after approximately $40 \mathrm{k}$-h of thruster operation, the same as seen in the multiple-simulation case. Figure 10 plots the final geometries for both single-simulation and multiple-simulation results. The geometries are very similar, although the multiple-simulation 
result is smoother because of the erosion rates being redistributed as some parts of the grid are worn away. In other words, CEX ions are more likely to erode geometry that sticks out, wearing it down until the ions can impact on other regions. The use of multiple simulations allows this redistribution because the erosion rates are updated as the geometry changes. The single-simulation case does not vary the erosion rates, and so no such redistribution can occur.

\section{Dynamic Erosion Results}

Finally, erosion is modeled using dynamic erosion of the ion optics. In this case, the simulation begins with the initial geometry and initializes the flow using this geometry. Once the flow is initialized, dynamic erosion is enabled. Whenever an ion impacts on an optics surface, the number of eroded molybdenum atoms is calculated. These atoms are removed from the optics cell, and if all of the atoms are removed from a cell that cell is no longer considered an ion-optics cell. Redeposition of sputtered grid material is not simulated here.

The simulation time step is on the order of $1 \times 10^{-10} \mathrm{~s}$, and the dynamic erosion is performed for 100,000 iterations, giving a total simulated time on the order of $0.01 \mathrm{~ms}$. To allow simulation of thruster life, the number of atoms in an optics cell must be scaled. This scaling factor is simply the amount of time the simulation actually models divided by the thruster erosion time. In this simulation, the thruster erosion time is set to $50,000 \mathrm{~h}$, and so the scaling factor is approximately $3 \times 10^{-13}$.

For a better comparison to the multiple- and single-simulation results, the dynamic erosion algorithm is restricted such that downstream face erosion does not occur or is mitigated. This is accomplished by not eroding material when an ion impacts on the downstream face of the grid. However, this still allows impacts on the downstream side of any eroded geometry or the cusp.

This method produces results very close to the multiple- and single-aperture simulation erosion. The eroded grid geometry and potential field after 15,30 , and $45 \mathrm{k}$-h of erosion are plotted in
Fig. 11. As before, electron backstreaming is assumed to occur when the $7.5-\mathrm{V}$ contour is connected between the upstream and downstream regions. In this case, backstreaming occurs after $45,000 \mathrm{~h}$ of erosion. Note that the downstream half of the grid is systematically chamfered by CEX ions from the downstream region. Although erosion is not allowed by these ions on the actual downstream face, they do contribute to erosion in other areas.

As shown by Fig. 10, the dynamic erosion geometry at which backstreaming occurs is very similar to the previous results. One difference is that this result is reached after approximately $45,000 \mathrm{~h}$ of erosion, rather than the $40,000 \mathrm{~h}$ needed in the multiple- and single-simulation cases. This difference most likely occurs because the geometry simulated in the dynamic erosion case depends on the mesh of that domain. In the multiple- and singe-erosion simulations, the true geometry is eroded using the simulated erosion rates. As the first two upstream points in Fig. 10 show, the dynamic erosion case must erode more material in some areas where the mesh does not exactly match the true grid geometry. Also, the potential field for the dynamic erosion case is a snapshot-it is the potential for only one iteration. The other cases are able to average the potential over many iterations to find the minimum centerline potential. Thus, the potential in the dynamic case can involve some statistical fluctuations.

Each type of prediction has advantages and disadvantages: multiple simulations require a large amount of computation time, whereas a single simulation gives very irregular geometry, even though the predicted life is the same. Dynamic simulation requires the same computation time as a single simulation, but the inaccuracy of the modeled geometry reduces the accuracy of the life prediction, although the final geometry is very similar to the multiple-simulation case.

\section{Summary}

The erosion predictions given here for the single- and multiplesimulation cases predict thruster failure caused by electron
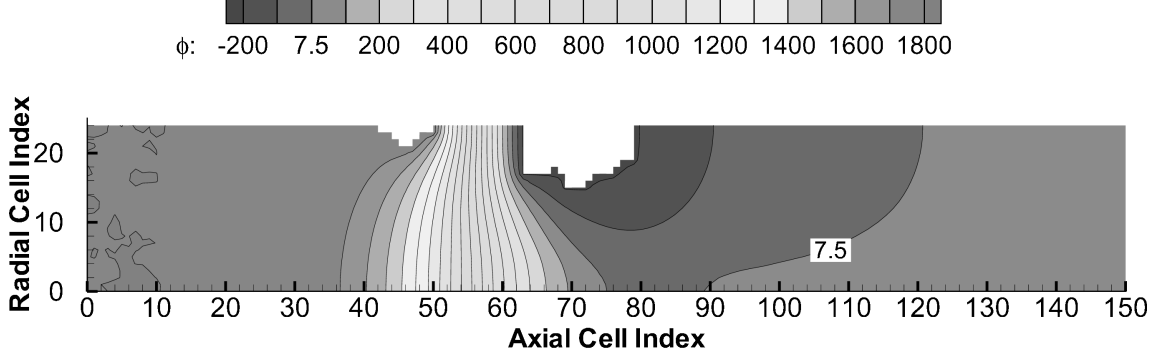

a) 15,000 hours of erosion

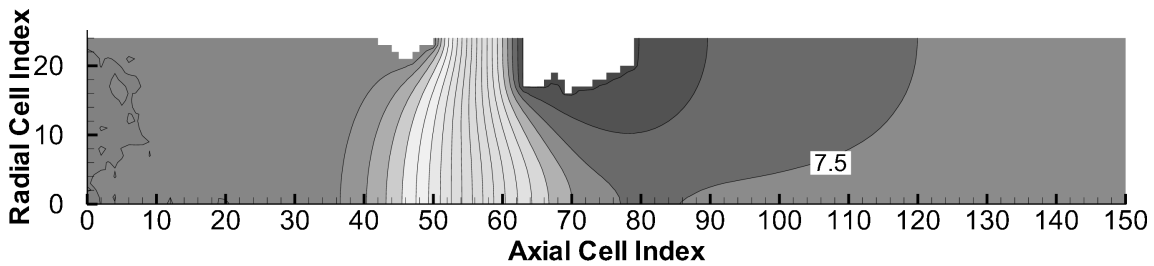

b) 30,000 hours of erosion

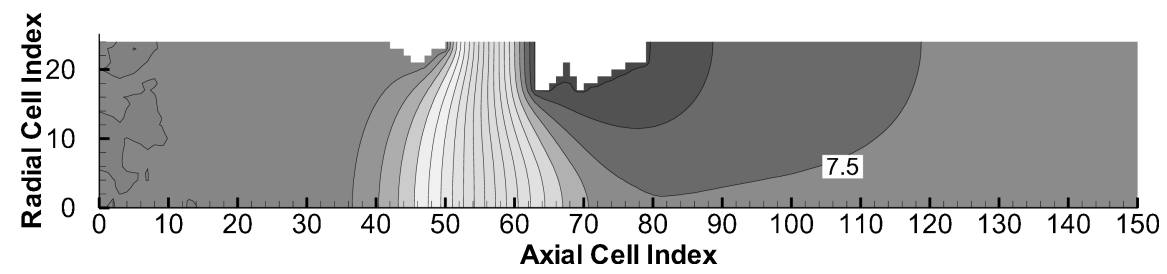

c) 45,000 hours of erosion

Fig. 11 Potential field contours as the accelerator grid is eroded dynamically, without downstream face erosion. Dimensions are in volts. The 7.5-V contour is labeled-when the downstream contour connects with the upstream contour, electron backstreaming will occur. After approximately $45,000 \mathrm{~h}$ of erosion, backstreaming begins. 
backstreaming after approximately $40,000 \mathrm{~h}$ of thruster operation at the maximum operating point. This corresponds to $845 \mathrm{~kg}$ of propellent throughput. As already described, this analysis does not account for the potential structural failure as a result of erosion on the downstream face. However, it is apparent that erosion rates from a single simulation are sufficient to predict the same electron backstreaming behavior given by multiple and dynamic simulations.

This model has been used previously to predict electron backstreaming. In previous simulations, ${ }^{12}$ the backstreaming limit was reached after approximately the same thruster operating time. Results for a three-dimensional model ${ }^{7}$ predict thruster failure much sooner, after a propellant throughput of $625 \mathrm{~kg}$. However, this estimate is for an accelerator grid potential of $-250 \mathrm{~V}$ and is caused by structural failure, not electron backstreaming. These results define thruster failure caused by structural failure as the point at which $50 \%$ of the grid material has been eroded. At an accelerator grid potential of $-200 \mathrm{~V}$, the estimate increases to $805 \mathrm{~kg}$ of throughput, much closer to the result just given, although end of life is again caused by structural failure in this case. The rapid erosion of the downstream face in the dynamic erosion simulation supports this prediction. Predictions based on experimental data give failure after $750 \mathrm{~kg}$ of throughput, ${ }^{2}$ also as a result of structural failure. In this case, structural failure is defined as the point at which the grooves will erode through the grid.

\section{Conclusions}

PIC simulation of several ion-optics apertures at different radii on the thruster surface provides a more accurate picture of thruster performance than simply using results from a single simulation. The model described here is capable of accurately simulating performance of the NEXT ion engine. Accelerator grid barrel erosion is thought to be modeled correctly. Erosion predictions from the measured profilometry data and the simulation results suggest that the experimentally measured current might be too high.

Three methods can be used to estimate the life of the thruster before the onset of electron backstreaming caused by aperture enlargement. The use of a single simulation, multiple simulations, and dynamic erosion all predict that the NEXT ion engine will encounter electron backstreaming after approximately $40,000 \mathrm{~h}$ of operation or $845 \mathrm{~kg}$ of xenon propellant throughput.

\section{Acknowledgments}

Support for this work is provided in part by NASA John H. Glenn Research Center at Lewis Field Grant NAG3-2497 with Jon Van Noord as the technical monitor. Support is also provided by a Michigan Space Grant Consortium Graduate Student Fellowship.

\section{References}

${ }^{1}$ Brophy, J. R., Brinza, D. E., Polk, J. E., Henry, M. D., and Sengupta, A., “The DS1 Hyper-Extended Mission,” AIAA Paper 2002-3673, July 2002.

${ }^{2}$ Soulas, G. C., Kamhawi, H., Patterson, M. J., Briton, M. A., and Frandina, M. M., "NEXT Ion Engine 2000 Hour Wear Test Results," AIAA Paper 2004-3791, July 2004

${ }^{3}$ Kamhawi, H., Soulas, G. C., Patterson, M. J., and Frandina, M. M., "NEXT Ion Engine 2000 Hour Wear Test Plume and Erosion Results," AIAA Paper 2004-3792, July 2004.
${ }^{4}$ Soulas, G. C., Haag, T. W., and Patterson, M. J., "Performance Evaluation of $40 \mathrm{~cm}$ Ion Optics for the NEXT Ion Engine," AIAA Paper 2002-3834, July 2002 .

${ }^{5}$ Malone, S. P., and Soulas, G. C., "Computational Ion Optics Design Evaluations," AIAA Paper 2004-3784, July 2004.

${ }^{6}$ Anderson, J. R., Katz, I., and Goebel, D., "Numerical Simulation of TwoGrid Ion Optics Using a 3D Code," AIAA Paper 2004-3782, July 2004.

${ }^{7}$ Farnell, C. C., Williams, J. D., and Wilbur, P. J., "NEXT Ion Optics Simulation Via ffx," AIAA Paper 2003-4869, July 2003.

${ }^{8}$ Kafafy, R., and Wang, J., "Whole Ion Optics Simulations of a Subscale Gridlet Using a Hybrid-Grid IFE-PIC Code," AIAA Paper 2004-3783, July 2004

${ }^{9}$ Okawa, Y., Hayakawa, Y., and Kitamura, S., "Three-Dimensional Divergence Characteristics of Ion Beamlets in an Ion Thruster," AIAA Paper 2004-3785, July 2004.

${ }^{10}$ Crofton, M. W., and Boyd, I. D., "The Origins of Accelerator Grid Current: Analysis of T5-Grid Test Results," AIAA Paper 99-2443, June 1999.

${ }^{11}$ Emhoff, J. W., and Boyd, I. D., "A Numerical Study of Neutralization and Sputtering Processes in the NSTAR Thruster," AIAA Paper 2002-4259, July 2002

${ }^{12}$ Emhoff, J. W., and Boyd, I. D., "Grid Erosion Modeling of the NEXT Ion Thruster Optics," AIAA Paper 2003-4869, July 2003.

${ }^{13}$ Emhoff, J. W., and Boyd, I. D., "Progress in NEXT Ion Optics Modeling," AIAA Paper 2004-3786, July 2004.

${ }^{14}$ Emhoff, J. W., "Simulation of Ion Optics Using Particle-in-Cell and Treecode Methods," Ph.D. Dissertation, Aerospace Engineering Dept., Univ. of Michigan, Ann Arbor, May 2005.

${ }^{15}$ Birdsall, C. K., and Langdon, A. B., Plasma Physics via Computer Simulation, Institute of Physics Publishing, Boca Raton, FL, 1991.

${ }^{16}$ Bird, G. A., Molecular Gas Dynamics and the Direct Simulation of Gas Flows, Oxford Univ. Press, Oxford, England, U.K., 1994.

${ }^{17}$ Dalgarno, A., McDowell, M. R. C., and Williams, A., "The Mobilities of Ions in Unlike Gases," Royal Society of London Philosophical Transactions Series A, Vol. 250, No. 982, 1958, pp. 411-425.

${ }^{18}$ Polk, J. E., Moore, N. R., Newlin, L. E., Brophy, J. R., and Ebbeler, D. H., "Probabilistic Analysis of Ion Engine Accelerator Grid Life," Proceedings of the International Electric Propulsion Conference, Paper 93-176, Sept. 1993.

${ }^{19}$ Rosenberg, D., and Wehner, G. K., "Sputtering Yields for Low Energy $\mathrm{He}+, \mathrm{Kr}+$, and $\mathrm{Xe}+$ Ion Bombardment," Journal of Applied Physics, Vol. 33, No. 5, 1962, pp. 1842-1845.

${ }^{20}$ Soulas, G. C., Domonkos, M. T., Kamhawi, H., and Patterson, M. J., "Status of the NEXT Ion Engine Wear Test," AIAA Paper 2003-4863, July 2003.

${ }^{21}$ Weijsenfeld, C. H., Hoogendoorn, A., and Koedam, M., "Sputtering of Polycrystalline Metals by Inert Gas Ions of Low Energy (100-1000 eV)," Physica, Vol. 27, No. 8, 1961, p. 763.

${ }_{22}$ Bhattacharjee, J., Zhang, J., Shutthanandan, V., Ray, P. K., Shivaparan, N. R., and Smith, R. J., "Application of Secondary Neutral Mass Spectrometry In Low-Energy Sputtering Yield Measurements," Nuclear Instruments and Methods in Physics Research B, Vol. 129, No. 1, 1997, pp. 123-129.

${ }^{23}$ Duchemin, O. B., "An Investigation of Ion Engine Erosion by Low Energy Sputtering," Ph.D. Dissertation, Graduate Aeronautical Laboratories, California Inst. of Technology, Pasadena, CA, March 2001.

${ }^{24}$ Kolasinski, R., "Oblique Angle Sputtering Yield Measurements for Ion Thruster Grid Materials," AIAA Paper 2005-3526, July 2005.

${ }^{25}$ Doerner, R. P., Whyte, D. G., and Goebel, D. M., "Sputtering Yield Measurements During Low Energy Xenon Plasma Bombardment," Journal of Applied Physics, Vol. 93, No. 9, 2003, pp. 5816-5823.

${ }^{26}$ Wang, J., Polk, J., Brophy, J., and Katz, I., "Three-Dimensional Particle Simulations of Ion-Optics Plasma Flow and Grid Erosion," Journal of Propulsion and Power, Vol. 19, No. 6, 2003, pp. 1192-1199. 